\title{
Comparing deuterium retention in tungsten films measured by temperature programmed desorption and nuclear reaction analysis
}

\author{
P. Wang, W. Jacob*, L. Gao, T. Dürbeck, T. Schwarz-Selinger \\ Max-Planck-Institute für Plasmaphysik, EURATOM Association, Boltzmannstr.2, 85748 Garching, Germany
}

\begin{abstract}
:
Tungsten (W) films with thicknesses ranging between 1 and $12 \mu \mathrm{m}$ deposited by magnetron sputtering on silicon substrates were used as a model system for comparing the deuterium (D) retention measured by both temperature programmed desorption (TPD) and nuclear reaction analysis (NRA). Samples were loaded with deuterium ex-situ with an ECR plasma at 370 and $600 \mathrm{~K}$ with an energy of $38 \mathrm{eV}$ per deuteron. To avoid diffusion of D into the silicon substrate and to increase adhesion a copper interlayer was applied. The results show that all implanted $\mathrm{D}$ atoms were retained exclusively in the $\mathrm{W}$ films. The distribution of $\mathrm{D}$ is homogenous throughout the $\mathrm{W}$ layer with an atomic fraction of $3 \pm 0.4 \times 10^{-3}$. With increasing $\mathrm{W}$ thickness the D profile extends to correspondingly larger depths with practically identical D concentration. For W films with a thickness lower than the NRA information depth of about $8 \mu \mathrm{m}$ the total retained D amount measured by TPD and NRA is in excellent agreement. As expected, for films thicker than the NRA information depth, TPD deviates from NRA.
\end{abstract}

Keywords: Tungsten; Deuterium retention, Nuclear reaction analysis Thermal desorption spectroscopy

PACS: 52.40.Hf, 79.20.Rf, 25.55.-e, 28.52.Fa

\begin{tabular}{|c|c|}
\hline $\begin{array}{l}\text { Published in: } \\
\text { doi: }\end{array}$ & $\begin{array}{l}\text { Nuclear Instruments and Methods in Physics Research B } 300 \text { (2013) 54-61. } \\
\text { http://dx.doi.org/10.1016/j.nimb.2013.01.057 }\end{array}$ \\
\hline Submitted: & 14.12 .2012 \\
\hline Accepted: & 01.02 .2013 \\
\hline Available online: & 16.02 .2013 \\
\hline
\end{tabular}

\footnotetext{
* Corresponding author. Fax:+49-3299-1504, Tel:+49-3299-2618.

E-mail address: wolfgang.jacob@ipp.mpg.de
} 


\section{Introduction}

Hydrogen plays an important role in the microstructure of various amorphous hydrogenated materials, such as e.g. amorphous hydrogenated silicon (a-Si:H) [1], carbon (a$\mathrm{C}: \mathrm{H})[2,3]$ and boron $(\mathrm{a}-\mathrm{B}: \mathrm{H})$ films [4]. Consequently, the quantitative determination of the hydrogen content is an important prerequisite for the understanding of the microscopic structure of these materials. In these amorphous materials the hydrogen content is typically distributed homogenously throughout the films but may vary significantly depending on deposition conditions. Typically it is of the order of a few to a few ten percent, so that the determination of the $\mathrm{H}$ content by, e.g., ion beam methods is no major problem.

On the other hand, in experiments with magnetically confined high temperature hydrogen plasmas such as investigated in controlled thermonuclear fusion research, the vessel walls are bombarded with hydrogen isotope ions $(\mathrm{H}, \mathrm{D}$, and $\mathrm{T})$ with high fluxes $\left(10^{20}-10^{24} \mathrm{~m}^{-2} \mathrm{~s}^{-1}\right)$ at energies between a few $\mathrm{eV}$ and $\mathrm{keV}$ [5-6]. This causes implantation and accumulation of hydrogen isotopes in the plasma-facing materials. In this respect, the evolution of the radioactive $\mathrm{T}$ inventory in the first wall, the permeation of $\mathrm{T}$ through the armour into the coolant and the thermo-mechanical stability after long term exposure are key parameters determining the applicability of a first wall material [5-6]. To understand and control the hydrogen isotopes balance and tritium retention in fusion devices it is important to be able to quantitatively analyse the retention of hydrogen isotopes in vessel wall materials, and to get information on the depth distributions and binding states of the hydrogen trapped in the vessel wall materials [5-9]. Due to its favourable physical properties, such as low erosion yield and high melting temperature, tungsten (W) is under consideration for use as plasma-facing material in the ITER divertor. Since the solubility of hydrogen in W is close to zero, hydrogen is only trapped at natural defects and radiation-induced defects with concentrations ranging from 0.1 atomic fraction near the surface to $10^{-7}$ atomic fraction throughout the bulk [10-12]. Because of this low concentration measuring hydrogen retention in $\mathrm{W}$ accurately is a challenging task [13]. An elegant way of doing this is using deuterium as implanted species. This hydrogen isotope can easily be distinguished from the ubiquitously present protium. Several analysis techniques can be applied for measuring the total amount or the depth distribution of deuterium in W. Nuclear reaction analysis (NRA) [14] can determine both, total amount and depth distribution. Secondary ion mass spectrometry (SIMS) [15] can provide depth distributions and temperature programmed desorption (TPD) (often also referred to as thermal desorption spectroscopy (TDS)) [16-24] allows measuring the total amount. TPD and NRA are frequently used to measure the deuterium retention in W.

In TPD the sample is heated with a linear temperature ramp and the released molecules are measured by quadrupole mass spectroscopy. With TPD the total deuterium amount in the sample can be quantified provided sufficiently high temperatures are applied to assure complete desorption. Typically the signals of the deuterium or deuterium-containing molecules are integrated over time and compared with calibrated gas leaks for determining absolute amounts [16-24]. However, TPD does not allow measuring the D depth distribution. Furthermore, if significant amounts of D are released in form of other molecules, e.g., as water or hydrocarbon molecules, if the samples have substantial oxygen or carbon contents, quantification might be hindered or even impossible. Hydrocarbons cannot be disentangled unambiguously from the spectra because of overlapping intensities in their cracking pattern and the sensitivity of adsorbing species such as water is nearly impossible to quantify [22-25].

The $\mathrm{D}\left({ }^{3} \mathrm{He}, \mathrm{p}\right) \alpha$ reaction has been successfully applied for depth profiling of $\mathrm{D}$ during the last three decades [26-33]. For tungsten, typically, the top $8 \mu \mathrm{m}$ can be analyzed using a 4.5 $\mathrm{MeV}^{3} \mathrm{He}$ incident beam [12-13]. Based on the deconvolution of the proton yields measured at different ${ }^{3} \mathrm{He}$ ion energies the deuterium depth profile up to $\mathrm{W}$ thicknesses of $8 \mu \mathrm{m}$ can be reasonably well deduced and the total retained $\mathrm{D}$ amount in this region can be determined by 
integrating the D profile over depth [27-32]. A drawback of NRA is the limitation of measurable depth. If $\mathrm{D}$ is retained in a $\mathrm{W}$ layer thicker than the NRA information depth of $8 \mu \mathrm{m}$ it cannot be analyzed by NRA. Consequently, the total D amount in bulk W measured by NRA should always be equal to or lower than the value measured by TPD. This assumption has been widely confirmed by different research groups [12, 29-31]. But in some cases opposite results were reported [23, 32-33]. These contradictory results can either be explained by experimental uncertainties, e.g. through problems in the quantification of the TPD signals, or the release of deuterium in the form of long chain hydrocarbons during TPD which cannot be well quantified [22].

In this work, well-defined $\mathrm{W}$ films with different thickness were deposited onto silicon substrates and loaded with deuterium under well-defined conditions. To avoid peeling of the tungsten films and to prevent deuterium diffusion into the silicon substrates a copper interlayer was used. Doing this we were able to produce deuterium-loaded W films with welldefined thickness. Using these layers as model systems both TPD and NRA were applied to measure the total retained deuterium amounts quantitatively.

\section{Experimental details}

Tungsten films with thicknesses from 1 to $12 \mu \mathrm{m}$ were deposited onto polished single crystalline (100) silicon wafers using a commercial sputtering device (Discovery ${ }^{\circledR} 18$, Denton). The purity of the used tungsten target material was $99.95 \mathrm{wt} \%$ (corresponding to about 99.79 at $\%$ ) with $\mathrm{Mo}, \mathrm{Fe}$ and $\mathrm{O}$ being the main impurities (lower than 50 weight ppm). The system was pumped down to the base pressure of less than $3 \times 10^{-5} \mathrm{~Pa}$ and the deposition was performed in argon atmosphere at $5 \times 10^{-1} \mathrm{~Pa}$. During $\mathrm{W}$ deposition the dc power applied to the tungsten sputter target was kept constant at $300 \mathrm{~W}$. At these settings the deposition rate of W is about $17 \mathrm{~nm} / \mathrm{min}$. Sample rotation during the deposition assured homogenous layer thickness. No extra substrate bias and heating or cooling was applied. A liquid $\mathrm{N}_{2}$ cold trap was attached to the deposition chamber to reduce the water partial pressure. Prior to deposition the silicon substrates were etched by an argon plasma at $-600 \mathrm{~V}$ bias for $2 \mathrm{~min}$ to remove the native oxide layer. All tungsten films investigated in this study were deposited onto the silicon substrates using an approximately $700 \mathrm{~nm}$ thick copper interlayer. The copper interlayer was deposited with $150 \mathrm{~W}$ dc power at $5 \times 10^{-1} \mathrm{~Pa}$. This yields a $\mathrm{Cu}$ deposition rate of $60 \mathrm{~nm} / \mathrm{min}$. Also here no substrate bias and heating or cooling was applied.

After deposition, a tactile profilometer (DEKTAK 8, Veeco) was applied to measure the thickness of the deposited films by scanning the step height between coated and uncoated area (area covered during deposition by a thin kapton tape). To determine the density of the deposited $\mathrm{W}$ films the mass change of a complete silicon wafer (4 inch in diameter) due to deposition of a $1.18 \mu \mathrm{m}$ thick $\mathrm{W}$ film was measured by a microbalance. Based on this weight change and the film thickness from profilometry a mass density of $17.5 \mathrm{~g} / \mathrm{cm}^{3}$ was derived which corresponds to $90 \%$ of the bulk W density.

For comparison, deuterium was also implanted in a rolled $0.8 \mathrm{~mm}$ thick polycrystalline tungsten sample (manufactured by PLANSEE Metall GmbH, 6600 Reutte, Austria) with a guaranteed purity of 99.97 wt.\%. The sample was mechanically polished to a mirror-like finish and cleaned in an ultrasonic bath with acetone. Prior to D implantation the sample was stress-relieved and degassed by heating for 2 hours at $1200 \mathrm{~K}$ in an ultra-high vacuum oven ( $\mathrm{p}$ $<5 \times 10^{-7} \mathrm{mbar}$ ). More details about the preparation of reference samples can be found in Ref. [29].

Deuterium implantation was carried out in the laboratory plasma experiment PlaQ. A basic description of PlaQ is given in [3,34,35]. In short, PlaQ consists of a stainless steel chamber and is equipped with a remote electron cyclotron resonance (ECR) plasma source. Microwaves $(2.45 \mathrm{GHz})$ are coupled into the vacuum vessel through a waveguide terminated 
by a quartz window located at the high B-field side. The magnetic field is created by a single magnetic coil. To decouple the plasma from the substrate, the plasma is confined in a metallic cage $150 \mathrm{~mm}$ in height and $140 \mathrm{~mm}$ in diameter. Particles can leave the cage in an axial direction through a hole in the bottom plate with a diameter of $55 \mathrm{~mm}$. A diverging plasma beam impinges perpendicularly onto the substrates which are located $100 \mathrm{~mm}$ below the cage exit. The energy of the ions impinging on the substrates can be varied by applying a dc or rf bias to the substrate electrode. In the experiments described here a dc bias was used. The sample holder and its support structure are made of copper and a boron nitride insulator is used in order to maximize the heat conductivity between the electrically isolated sample holder and the heat sink. To avoid contamination by copper all parts are coated with a $3 \mu \mathrm{m}$ thick magnetron sputtered tungsten film. Samples are tightly clamped to the holder by four molybdenum screws to optimize thermal contact. The temperature of the sample holder is stabilized using an open circuit thermostat with silicon oil. Temperatures are measured by a thermocouple attached to the sample holder from the back side. An infrared camera is used to observe the sample surface. The temperature measured by the IR camera is calibrated against the thermocouple temperature in steady state without plasma operation.

To ensure identical conditions in all implantation processes, the microwave output power for the ECR plasma was set to $144 \mathrm{~W}$ with a constant $\mathrm{D}_{2}$ gas pressure prior to plasma ignition of $1.0 \mathrm{~Pa}$ (gas flow $50 \mathrm{sccm}$ ). At floating potential, the total deuteron flux in the form of ions is $5.6 \times 10^{19} \mathrm{Dm}^{-2} \mathrm{~s}^{-1}$. The flux consists dominantly of $\mathrm{D}_{3}{ }^{+}$ions $(94 \%$ of the impinging ions corresponding to $97 \%$ of the impinging deuterons in form of ions) with minor contributions of $\mathrm{D}_{2}^{+}\left(2 \%\right.$ of the deuterons) and $\mathrm{D}^{+}$( $1 \%$ of the deuterons) [35]. The ion flux increases with increasing substrate bias [35]. In this article we refer to the energy per deuterium atom of the dominant molecular ion species as the ion energy. For example, if the implantation was performed at a dc substrate bias of $-100 \mathrm{~V}$ this results, together with the plasma potential of about $-15 \mathrm{~V}$ [35], in an ion energy of about $115 \mathrm{eV}$. This corresponds to a mean energy of about $38 \mathrm{eV}$ per deuteron for the dominant $\mathrm{D}_{3}{ }^{+}$ions. The deuteron flux for these settings is $9 \times 10^{19} \mathrm{Dm}^{-2} \mathrm{~s}^{-1}$ [35]. In the experiment described here deuterium implantation was carried out at a sample temperature of 370 and $600 \mathrm{~K}$

Rutherford backscattering spectrometry (RBS) was applied to measure the atomic areal density of the deposited films and to check the purity of the deposited layers. A beam of $4.0 \mathrm{MeV}^{4} \mathrm{He}$ was used at normal incidence and a scattering angle of $165^{\circ}$. A charge of $15 \mu \mathrm{C}$ was usually accumulated for one RBS spectrum. The total atomic areal density of the $1.18 \mu \mathrm{m}$ thick W film determined by RBS is $6.68 \times 10^{22}$ atoms $/ \mathrm{m}^{2}$, corresponding to a mass density of $17.4 \mathrm{~g} / \mathrm{cm}^{3}$. This value is in good agreement with the value determined from mass change and layer thickness $\left(17.5 \mathrm{~g} / \mathrm{cm}^{3}\right)$. Both measurements show that the density of as-deposited W film is about $10 \%$ lower than that of bulk W $\left(19.3 \mathrm{~g} / \mathrm{cm}^{3}\right)$. No traces of impurities were found in the RBS spectra. Unfortunately, the RBS technique is not very sensitive for detection of low $\mathrm{Z}$ impurities in the deposited tungsten films. Simulations for our experimental conditions with SIMNRA [36] show that oxygen and carbon would be visible in the RBS spectra for concentrations higher than about 5 at $\%$. So, RBS yields an upper limit for oxygen and carbon impurities of $5 \%$. Additional investigations by XPS (x-ray photoelectron spectroscopy) depth profiling show no indication of impurities in the bulk of the films. The sensitivity of the used XPS setup for impurities is below $1 \%$ so we can state that our as-deposited layers are pure tungsten films and impurity levels have to be lower than $1 \%$.

The amount of $\mathrm{D}$ retained in the samples was measured by NRA by means of the $\mathrm{D}\left({ }^{3} \mathrm{He}, \alpha\right) \mathrm{p}$ nuclear reaction two months after implantation. The D concentration within the near-surface layer (at depths up to about $0.3 \mu \mathrm{m}$ ) was determined at a ${ }^{3} \mathrm{He}$ energy of $0.69 \mathrm{MeV}$ by analyzing the emitted $\alpha$ particles with a surface barrier detector at the laboratory scattering angle of $102^{\circ}$ equipped with a rectangular slit reducing the solid angle to $9.16 \mathrm{msr}$. For determining the $\mathrm{D}$ concentration at larger depths, the energy of the analyzing beam of ${ }^{3} \mathrm{He}$ 
ions was varied from 0.69 to $4.5 \mathrm{MeV}$ depending on the thickness of $\mathrm{W}$ layer. In this work, the following ion beam energies were used in the analysis of the thickest $\mathrm{W}$ films: $0.69,1.2$, $1.8,2.4,3.2$, and $4.5 \mathrm{MeV}$. A charge of $10 \mu \mathrm{C}$ was usually accumulated for one NRA spectrum. For 1 and $3 \mu \mathrm{m} \mathrm{W}$ films the maximum energies of the ${ }^{3} \mathrm{He}$ probing ions are 1.8 and $3.2 \mathrm{MeV}$, respectively. The high energy protons from the $\mathrm{D}\left({ }^{3} \mathrm{He}, \mathrm{p}\right)^{4} \mathrm{He}$ nuclear reaction were counted using a thick, large angle solid state detector at a scattering angle of $135^{\circ}$ equipped with a curved slit reducing the solid angle to $29.9 \mathrm{msr}$. In order to analyze the D concentration profile in the whole layer both SIMNRA [36] and NRADC [13] were used for the deconvolution of the NRA spectra measured at different ${ }^{3} \mathrm{He}$ ion energies. As input data for NRADC we use the $\alpha$ spectrum measured at $0.69 \mathrm{MeV}$ and all proton spectra measured at the different energies. Details about the data evaluation using NRADC can be found in Ref. [13], but some important issue are mentioned here: One of the key features of NRADC is that it not only finds the most probable D concentration profile but also chooses the most probable depth sampling. In particular, NRADC chooses the number of layers in the resulting depth profiles such that it minimizes the number of parameters required to describe the measured NRA data. It thus follows Occam's razor principle. As described in great detail in [13] this is done by starting with an initial depth sampling based on stopping power and NRA cross-section peak energies. After subdividing this initial depth sampling the profile is then refined further to finally yield the most probable depth profile and the respective confidence intervals for the D concentration in the individual layers. The error bars given in all depth profiles are only the statistical uncertainties determined by NRADC and thus do not describe the total uncertainty of the measurement. The total uncertainty of the measurement would include contributions from other parameters not estimated by NRADC during the optimization procedure like for instance the energy calibration, the current measurement, solid angle determination, and the used cross sections. In addition, the uncertainty in the depth spectra increases with increasing depth. For our experimental conditions the sensitivity of NRA for depths exceeding about 1$2 \mu \mathrm{m}$ in tungsten is about $1 \times 10^{-4} \mathrm{D}$. Values lower than that cannot be distinguished from the background noise. For the quantitative analysis we used the cross section published by Alimov [28]. The total amount of $\mathrm{D}$ retention was finally determined by integrating the $\mathrm{D}$ profile over the measured depth. Using $a-C: D$ films for calibration it has been shown earlier that the uncertainty for determination of D amounts by NRA is about $10 \%$ [16]. To check the performance of the detectors and to minimize the scatter in absolute $\mathrm{D}$ areal densities in our measurements a calibration sample of known D content was measured for $0.69 \mathrm{MeV}$ and at $2.4 \mathrm{MeV}$. The accuracy of the beam current measurement (typically $5 \%$ ) together with the counting statistics about 1 to $3 \%$ (counts depending on D content and energy) assures the accuracy of our measurements stays within this $10 \%$

After the NRA measurement the retained D amount of several selected samples was additionally measured by temperature programmed desorption (TPD) in the quartz tube of the TESS device. A basic description of TESS is given in [22]. The temperature response of the samples to the linear oven temperature ramp was calibrated in independent experiments by a thermocouple spot-welded to a tungsten sample of identical size and surface finish. The samples were heated up to a sample temperature of $1275 \mathrm{~K}$ with an oven heating rate of $15 \mathrm{~K} / \mathrm{min}$. This maximum temperature of $1275 \mathrm{~K}$ is high enough to ensure desorption of all retained $\mathrm{D}$ from the samples. The desorbed gases were measured with a quadrupole mass spectrometer (QMS). The secondary electron multiplier of the QMS was operated in single ion counting mode to minimize the background noise and to be able to apply Poisson statistics for determining the accuracy. Selected mass channels between 2 and 44 amu were recorded as a function of time (so called multiple ion detection mode of the QMS). The following 15 mass channels were recorded: 1, 2, 3, 4, 12, 14, 16, 17, 18, 19, 20, 28, 32, 40, and 44 amu. Some of them are just measured to assess background emission and to check that no other species are released. 
For the quantitative analysis the QMS signals for $\mathrm{HD}, \mathrm{D}_{2}$ and $\mathrm{CD}_{4}$ were calibrated. The following procedure was applied for calibration: Calibration gases were introduced through an aperture $80 \mu \mathrm{m}$ in diameter and guided through a capillary into the device. The conductance of the capillary is much higher than that of the aperture, so that the flux is only determined by the aperture. The conductance of this aperture was determined experimentally for the different gases using the method suggested by Tschersich [37]. A given volume of unknown size at the high pressure side of the aperture was filled with the calibration gas to a pressure of the order of $10^{-2}-10^{-1} \mathrm{~Pa}$. The pressure on the high pressure side was measured with a spinning rotor gauge (rated accuracy of 1-10\% depending on the pressure range). After closing the input valve the gas was purged into the high vacuum system. The exponential decay of the QMS signal and pressure reading of the pressure gauge was recorded as a function of time. The same procedure was repeated adding a known volume on the high pressure side. From these two measurements the gas flow can be determined and the uncertainty is only given by the accuracy of the pressure measurement and the volume measurement which is lower than $2 \%$. The QMS signal was then calibrated using this set-up and applying count rates typically achieved during a TPD experiment (between 500 and 20000 counts per second in the desorption maximum). A certain constant gas flow was introduced into the UHV chamber and the QMS signal was measured until the QMS reading was constant. This procedure was repeated for five different flow levels and the linearity was checked. The calibration factors for HD and $\mathrm{D}_{2}$ are $(3.5 \pm 0.14) \times 10^{9}$ molecules per count, and $(3.8 \pm 0.15) \times 10^{9}$ molecules per count, respectively. The error bars are determined by consecutive calibration measurements and are governed by the stability of the detector rather than by determination of the flow. This calibration procedure was additionally cross checked for $\mathrm{D}_{2}$ in an independent measurement using a calibrated leak (Laco Technologies). The certified flow of this leak was $1.22 \times 10^{14}$ molecules/s with a rated accuracy of $\pm 4.6 \%$. The flow determined for the same QMS reading according to the above calibration procedure was $1.39 \times 10^{14}$ molecules/s. Consequently, we assume that the QMS calibration for $\mathrm{HD}, \mathrm{H}_{2}$ and $\mathrm{D}_{2}$ has an uncertainty of less than $10 \%$.

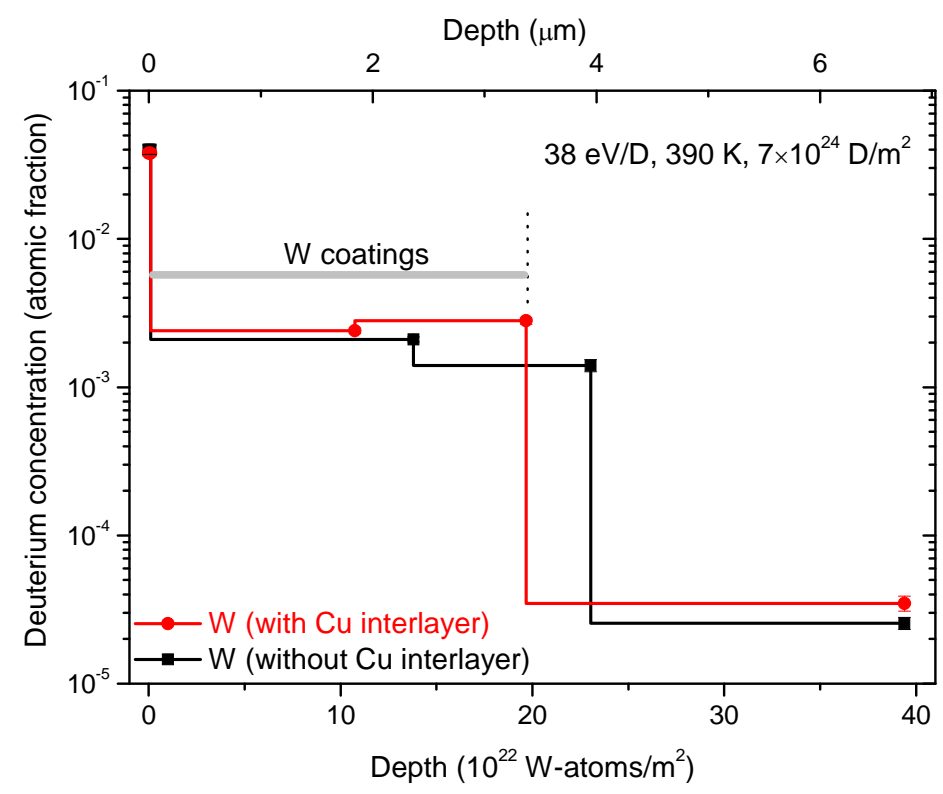

Fig. 1: Deuterium depth profiles of $3.4 \mu \mathrm{m}$ thick $W$ films deposited on silicon substrates with and without copper interlayer. Deuterium implantation was performed at $390 \mathrm{~K}$ with $38 \mathrm{eV/D}$ incident energy and a fluence of $7 \times 1024 \mathrm{D} / \mathrm{m} 2$. The interface between the $W$ film and the substrate is marked in the figure by the dashed line. 


\section{Results and discussion}

\subsection{Effect of copper interlayer}

In a first set of experiments, the $\mathrm{W}$ film was directly deposited onto silicon without any interlayer; however, we found that the $\mathrm{W}$ film often delaminated during $\mathrm{D}$ implantation or during the storage period afterwards. Moreover, NRA measurements from intact samples - to be presented below-showed that deuterium atoms diffuse through the whole tungsten layer and partly penetrate into the silicon substrate thus contributing to the total deuterium retention. To avoid these problems $700 \mathrm{~nm}$ thick copper films were deposited onto the silicon substrates as an interlayer. Such copper interlayers have been used earlier in our laboratory to avoid peeling of magnetron-sputtered $\mathrm{W}$ films [38].

Fig. 1 shows the deuterium depth profiles of about $3.4 \mu \mathrm{m}$ thick $\mathrm{W}$ films with and without copper interlayer. In this case, $3.4 \mu \mathrm{m}$ thick $\mathrm{W}$ films were deposited on two different silicon substrates (with or without $\mathrm{Cu}$ interlayer) simultaneously. D implantation was performed simultaneously in both samples at a sample holder temperature of $390 \mathrm{~K}$ with $38 \mathrm{eV} / \mathrm{D}$ incident particle energy and a fluence of $7.0 \times 10^{24} \mathrm{D} \mathrm{m}^{-2}$. Please note that in contrast to all following experiments which were performed at a temperature of $370 \mathrm{~K}$ this implantation was carried out at $390 \mathrm{~K}$. In Fig. 1 the deuterium concentration deduced by NRADC is plotted as function of depth with the $\mathrm{W}$ areal density as unit (bottom axis). Additionally, the corresponding $\mathrm{W}$ thickness (using the measured mass density, see Section 2) is plotted on the top axis. In general, the two samples show very similar deuterium depth profiles. For the W film deposited with $\mathrm{Cu}$ interlayer, a high deuterium concentration is found in a thin nearsurface layer (about $16 \mathrm{~nm}$ thick) with $4 \%$ deuterium concentration and a nearly constant concentration of about $0.26 \%$ extends throughout the remaining $\mathrm{W}$ film. With respect to the surface layer we have to add the following remark: The thickness of this layer is determined by the depth resolution of the $\alpha$ spectrum measured at $0.69 \mathrm{MeV}$. This resolution at the surface is $16 \mathrm{~nm}$. Correspondingly, the given $\mathrm{D}$ concentration of $4 \%$ is the mean $\mathrm{D}$ concentration in this layer. It could very well be that the real thickness of the surface layer is thinner than the experimentally achievable depth resolution. In that case the local $\mathrm{D}$ concentration in that layer would be correspondingly higher.

For the $\mathrm{W}$ film directly deposited on silicon (without $\mathrm{Cu}$ interlayer) also a comparably high D surface concentration is found and the deuterium concentration in the bulk W film is about $0.18 \%$. A notable difference for the two cases appears only at the interface. For the W film deposited onto silicon with the $\mathrm{Cu}$ interlayer, the deuterium concentration drops at the interface between $\mathrm{W}$ and $\mathrm{Cu}$ by two orders of magnitude from about $2 \times 10^{-3}$ to $2 \times 10^{-5}$. As explained in Section 2 the latter value is already below the detection limit of the NRA method and is therefore equivalent to zero. We interpret these data such that the diffusion of deuterium into silicon is blocked by this magnetron-sputtered copper layer and all retained deuterium atoms are within the $\mathrm{W}$ layer. On the contrary, for the $\mathrm{W}$ film deposited directly onto the silicon substrate the deuterium depth profile obviously exceeds the $\mathrm{W} / \mathrm{Si}$ interface and penetrates up to a few hundred $\mathrm{nm}$ into the silicon. We assume that this is due to the diffusion and trapping of $\mathrm{D}$ in silicon in the region close to the interface. Clearly, we do not anticipate a step-like depth profile of deuterium in the silicon sample. The step resulting from the NRADC evaluation is owing to the low depth resolution of NRA in this large depth which does not allow a better reconstruction of the profile. Nevertheless, the measured proton spectra for this sample cannot be fitted well without assuming some D being present in the silicon substrate close to the interface.

One interesting observation is that the $\mathrm{D}$ concentration in the $\mathrm{W}$ film with the $\mathrm{Cu}$ interlayer shows a $30 \%$ higher value than in the $\mathrm{W}$ film without $\mathrm{Cu}$ interlayer. This could be attributed to a change of the microstructure of the $\mathrm{W}$ film due to the introduction of the $\mathrm{Cu}$ 
interlayer, which could affect the nucleation and growth process of the deposited W film although both $\mathrm{W}$ films were deposited in same batch.

In previous experiments, $700 \mathrm{~nm}$ thick copper layers deposited onto silicon substrates applying identical deposition conditions as for the $\mathrm{Cu}$ films used in this study were used as samples for measuring the sputtering yield after deuterium plasma exposures at different dc and rf bias voltages [39]. These experiments were performed in order to calibrate the deuterium ion flux in the PlaQ device at different rf bias voltages. Simultaneously the deuterium retention in these sputtered $\mathrm{Cu}$ films was measured by NRA. The results (not presented here) show that for all investigated bias voltages and fluences only about one monolayer deuterium is retained in the near-surface region corresponding to the implantation zone. This proved that deuterium retention in $\mathrm{Cu}$ for our implantation conditions is insignificant. This observation can be attributed to the negligible solubility and low diffusivity of hydrogen in copper [40]. Consequently, the copper layer deposited onto the silicon substrates acts as a diffusion barrier which blocks the diffusion of deuterium into the silicon. For all samples investigated in the following a $700 \mathrm{~nm}$ thick copper interlayer was applied and only the thickness of the $\mathrm{W}$ films was varied from about 1 to $12 \mu \mathrm{m}$. For convenience, the notation being used throughout the remaining text to describe a layer system with an $\mathrm{x} \mu \mathrm{m}$ thick tungsten film on top is $\mathrm{W}-\mathrm{x}$.

\subsection{Deuterium retention measured by NRA}

Deuterium retention in $\mathrm{W}$ films as a function of incident fluence was thoroughly investigated in preceding experiments. The results show that for the applied experimental conditions the retained D amount in thin $\mathrm{W}$ films initially quickly increases with increasing fluence (roughly proportional to fluence ${ }^{0.7}$ ) up to fluences of about $3 \times 10^{23} \mathrm{D} / \mathrm{m}^{2}$. For higher fluences it continues increasing but much more slowly (roughly proportional to fluence ${ }^{0.2}$ ). In the present work all $\mathrm{W}$-x layer systems were implanted with an incident fluence of $6 \times 10^{24} \mathrm{D} / \mathrm{m}^{2}$. Fig. 2 shows the deuterium depth profiles of $\mathrm{W}-\mathrm{x}$ layer systems implanted at $38 \mathrm{eV} / \mathrm{D}$ incident energy with a fluence of $6 \times 10^{24} \mathrm{D} / \mathrm{m}^{2}$. All implantations in this section were performed at $370 \mathrm{~K}$.

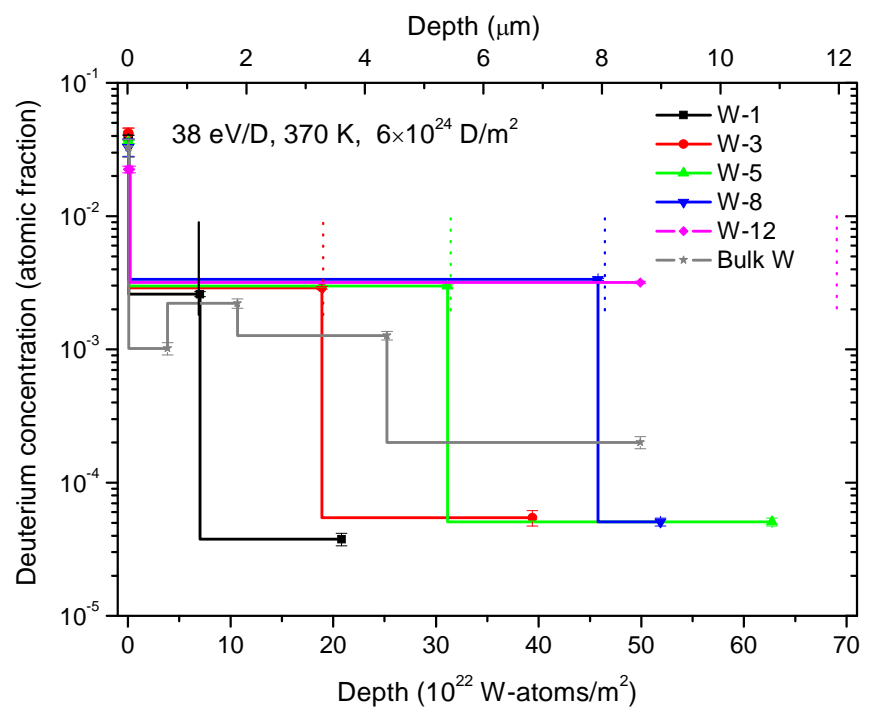

Fig. 2: Deuterium depth profiles in $W$-x layer systems after deuterium implantation at $38 \mathrm{eV} / \mathrm{D}$ incident energy with a fluence of $6 \times 1024 \mathrm{D} / \mathrm{m} 2$. In all cases, the thickness of the Cu interlayer was $700 \mathrm{~nm}$. The numbers in the notation correspond to the thickness of W layers determined by $R B S$ and profilometry. The interfaces between the $W$ layer and the copper were marked according to RBS (solid line) and profilometry measurements (dashed lines). 
For $\mathrm{W}-1$ the interface between $\mathrm{W}$ and $\mathrm{Cu}$ is marked (solid line) according to the RBS results. For thicker $\mathrm{W}$ films the thickness of the $\mathrm{W}$ films cannot be determined from the RBS measurements, because the stopping of the probing $4 \mathrm{He}$ particles in $\mathrm{W}$ is too high such that $4 \mathrm{He}$ particles backscattered from the W-Cu interface cannot leave the sample. But because the film thickness has been measured after deposition by profilometry and since the density has been determined (see Section 2) the position of the W-Cu interface on the areal density scale in Fig. 2 (in units of atoms per $\mathrm{m} 2$ ) can be reasonably well deduced. The exact thicknesses of the investigated $\mathrm{W}$ layers measured by profilometry are $1.1,3.3,5.3,7.8$, and $11.8 \mu \mathrm{m}$, respectively. The positions of the corresponding interfaces for the thicker films are marked by dashed lines in Fig. 2.

All samples show a deuterium concentration at the surface which is one order of magnitude higher than the bulk concentration. This surface layer is $16 \mathrm{~nm}$ thick and has a deuterium concentration between 3 and $4 \%$. With respect to thickness and D concentration of this layer see the comments made in Section 3.1. In the bulk of the film the deuterium profile is flat with an atomic fraction of about $3 \pm 0.4 \times 10^{-3}$. With increasing W thickness the deuterium profiles extend to a larger depth with practically identical D concentration. The D concentration in the bulk of sample W-3 is slightly higher (20\%) compared with the sample shown in Fig. 1. This is attributed to the different temperatures during these implantations. In all cases shown in Fig. 2 a sharp drop of the D concentration is observed at the interface between $\mathrm{W}$ and $\mathrm{Cu}$. Beyond this interface the deuterium concentration drops by two orders of magnitude to $10^{-5}$, which is equivalent to the sensitivity limit of the analysis method. The integrated deuterium amount in this low D concentration region behind the interface would contribute 1 to $3 \%$ to the total retained D amount. For the further analysis this contribution is ignored because we assume that the $\mathrm{D}$ concentration in the copper interlayer and the underlying $\mathrm{Si}$ bulk is negligible. The NRA results clearly prove that in these $\mathrm{W}-\mathrm{x}$ layer systems the retained deuterium is completely contained in the W layer only.

For comparison a D depth profile in a stress-relieved polycrystalline $\mathrm{W}$ reference sample is also shown in Fig. 2. D implantation into the $\mathrm{W}$ bulk sample was carried out under the identical implantation conditions as for the W-x layer systems. Deuterium retention in bulk tungsten is inhomogeneous in depth. Again a D-rich layer as for the magnetron-sputtered films is found at the surface. In the depth range up to about $4 \mu \mathrm{m}$ the $\mathrm{D}$ concentration in bulk $\mathrm{W}$ is about a factor of 3 lower than in the films and for larger depths it is about one order of magnitude lower than in the films. Since all deuterium retention measurements were performed two months after implantation, all detected deuterium atoms should be retained in natural defects $[10,12,24]$. The higher deuterium retention in the $\mathrm{W}$ films means that the defect density in these sputter-deposited films is higher than in bulk W.

One interesting point is that apart from the D-rich surface layer the D distributes homogenously in the W film, and with increasing W film thickness D extends to deeper layer with constant concentration. This D diffusion behaviour is quite different from that in bulk W $[12,29]$. Currently we don't have a reasonable explanation for that. To understand the difference more work is required and further systematic studies are underway. 


\subsection{Deuterium retention measured by TPD}

After the NRA measurement the retained D amount was measured by TPD for several selected samples. In all cases, samples were heated to a sample temperature of $1275 \mathrm{~K}$ with a ramp of $15 \mathrm{~K} / \mathrm{min}$ and held at this high temperature for $15 \mathrm{~min}$. Typical spectra-presented here are data for W-8 implanted at $38 \mathrm{eV} / \mathrm{D}$ with a fluence of $6 \times 10^{24} \mathrm{D} / \mathrm{m}^{2}$-for mass channels 3, 4, 19 and 20 are shown in Fig. 3. The QMS raw signals are plotted as a function of elapsed time (left-hand scale). In addition, the oven and sample temperature ramps are also shown (right-hand scale). Over the whole range the oven temperature is significantly higher than the true sample temperature. At low oven temperature the difference is more than $100 \mathrm{~K}$. For temperatures larger than about $600 \mathrm{~K}$ the offset reduces to about $70-80 \mathrm{~K}$ remaining constant at this value up to the end temperature. $\mathrm{D}_{2}$ desorption (4 amu) exhibits a sharp peak with a shoulder at higher temperature. $\mathrm{D}_{2}$ desorption starts at a sample temperature of about $400 \mathrm{~K}$, the temperature of the peak maximum is about $510 \mathrm{~K}$ and the shoulder is located at about $700 \mathrm{~K} . \mathrm{D}_{2}$ desorption reaches very low values at about $900 \mathrm{~K}$ and vanishes completely at about $1100 \mathrm{~K}$. Also during the holding time at the maximum temperature $\mathrm{D}_{2}$ release is negligible. The HD (3 amu) release spectrum extends over a larger temperature region, starting at about $450 \mathrm{~K}$ and extending to $1200 \mathrm{~K}$. The majority of HD is released below about $900 \mathrm{~K}$. Taking into account the calibration factors derived in Section 2 and the fact that HD carries only one deuterium atom while $\mathrm{D}_{2}$ carries two, the amount of deuterium released as $\mathrm{HD}$ is for W-8 as well as for all other investigated samples about $30 \%$. However, because we calibrated for HD this has no influence on the accuracy for the determination of the total D amount. The total amount of deuterium released from the samples was determined by integrating the QMS signal over time for masses 3 and 4. For W-8 the integrated QMS signal is $2.9 \times 10^{7}$ counts. Due to the large number of counts the error due to counting statistics is negligible. Even for the thinnest film (W-1) the integrated QMS signal is $4.1 \times 10^{6}$ counts and the corresponding standard deviation due to counting statistics is only $0.05 \%$. The total uncertainty is rather determined by the stability of the detector and the contribution of additional $\mathrm{D}$ containing species besides $\mathrm{HD}$ and $\mathrm{D}_{2}$. Apart from the signals at 3 and $4 \mathrm{amu}$ we find minor signals at mass channel 17, 19, and 20 that are attributed to molecules containing deuterium: According to their cracking pattern the two low intensity peaks at 19 and $20 \mathrm{amu}$ can be due to the release of $\mathrm{HDO}$ and $\mathrm{D}_{2} \mathrm{O}$. Please observe that these 2 signals are enlarged by a factor of 5 in Fig. 3. The intensity of the 20 amu peak is negligible meaning that very little $\mathrm{D}_{2} \mathrm{O}$ is formed during thermal release. $\mathrm{CD}_{4}$ can be excluded because of the expected cracking pattern. However, the 19 amu peak corresponding to HDO shows a clear peak in the low temperature region. While it is relatively straight forward to calibrate the $\mathrm{HD}$ and $\mathrm{D}_{2}$ signals using calibrated gas leaks, it is, in general, very hard to quantify water signals by mass spectrometry. Hydrogen molecules do not get permanently lost at the chamber walls and can therefore be transported via many wall collisions to the ioniser of the QMS. In contrary, water molecules have a significant, strongly temperature-dependent sticking coefficient and get permanently lost to the chamber walls. The dominant challenge to correctly quantify the water signal is to reasonably estimate the loss to the chamber walls. This challenge can in principle be overcome if all relevant surfaces which might be hit by water molecules are at sufficiently high temperature such that sticking is negligible. This is not the case in our set-up as in many others and therefore one cannot quantify the amount of deuterium released in form of HDO accurately. 


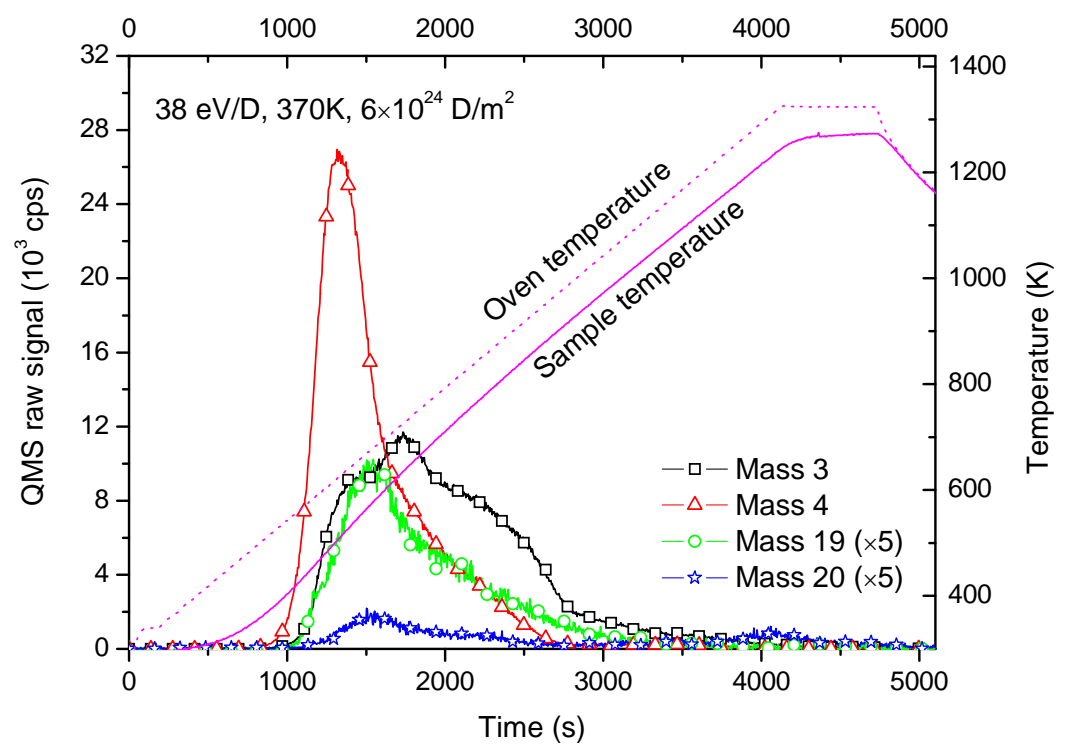

Fig. 3: QMS raw signals for mass channels 3, 4, 19 and 20 plotted as a function of time, the sample $W$-8 was implanted at $38 \mathrm{eV} / \mathrm{D}$ incident energy with a fluence of $6 \times 1024 \mathrm{D} / \mathrm{m} 2$. The oven heating rate is $15 \mathrm{~K} / \mathrm{min}$, the oven and sample temperatures are shown on the right hand scale. Note the intensities of mass channels 19 and 20 are enlarged by a factor of 5 .

In the following we try to estimate the possible contribution of the HDO signal (mass channel 19). For W-8 the integrated HDO QMS raw signal corresponds to about $10 \%$ of the $\mathrm{D}_{2}$ signal. The tabulated sensitivity factor of $\mathrm{H}_{2}$ is 0.44 and for $\mathrm{H}_{2} \mathrm{O}$ this value is 0.90 [41, 42]. Because we have no relative sensitivity factor for $\mathrm{HDO}$ we assume the same one as for $\mathrm{H}_{2} \mathrm{O}$. In total this result in an about $20 \%$ higher sensitivity of HDO compared with $\mathrm{D}_{2}$. Taking further into account that the HDO molecule contains only $1 \mathrm{D}$ atom while $\mathrm{D}_{2}$ has 2 the measured HDO signal would contribute about $2.1 \%$ to the total deuterium release. But due to the unknown loss of water to the chamber wall the actually released amount could be higher.

In addition to $\mathrm{D}_{2} \mathrm{O}$ and $\mathrm{HDO}$ a small signal is observed for mass channel 17 (not shown in Fig. 3). This signal we attribute to $\mathrm{CH}_{3} \mathrm{D}$. If we apply the same procedure to estimate the $\mathrm{D}$ release in form of $\mathrm{CH}_{3} \mathrm{D}$ as for $\mathrm{HDO}$ we get a possible contribution of $1.5 \%$.

Converting the signals for W-8 with the given sensitivities to D amounts we end up with $2.5 \times 10^{15} \mathrm{D} / \mathrm{cm}^{2}$ from $\mathrm{CH}_{3} \mathrm{D}, 3.5 \times 10^{15} \mathrm{D} / \mathrm{cm}^{2}$ from $\mathrm{HDO}$, and $0.55 \times 10^{15} \mathrm{D} / \mathrm{cm}^{2}$ from $\mathrm{D}_{2} \mathrm{O}$, respectively. This yields a relative contribution of these species to the total D release of $3.8 \%$. For thinner $\mathrm{W}$ samples the absolute $\mathrm{D}$ amounts from these species remain approximately the same independent of the $\mathrm{W}$ layer thickness such that the relative contributions increase. For $\mathrm{W}-1$ it amounts to $12.6 \%$. However, given the fact that these contributions do not change significantly with $\mathrm{W}$ layer thickness we attribute them to adsorbed layers on the sample surfaces. Therefore we do not add it to the total amounts.

The total amount of deuterium measured by TPD was only determined by integrating the signals of mass channels 3 and 4 over time. Because the release signals are practically zero before the highest temperature is reached, we are sure that all previously implanted deuterium is released during the TPD measurement. As a consequence, TPD measures the total amount of retained deuterium. The total uncertainty of the TPD results is mainly due to the detector stability and the neglect of the $\mathrm{HDO}, \mathrm{D}_{2} \mathrm{O}$ and $\mathrm{CH}_{3} \mathrm{D}$ signals and it is estimated to be lower than $10 \%$ even for the thinnest sample. It is also clear that by neglecting these contributions the $\mathrm{D}$ content is slightly but systematically underestimated. 
The shape of the TPD spectra is influenced by the trap energy and by the diffusion of D in the sample. Much work has been performed in the past to relate trap energies to the observed desorption temperatures $[12,18,21,26,30]$. However, due to differences of the investigated samples and the used experimental settings published data show some scatter. Generally, deuterium released at lower temperature is attributed to deuterium trapped in low-energy traps, such as grain boundaries and dislocations, while $\mathrm{D}$ released at higher temperature is attributed to $\mathrm{D}$ in high-energy traps, for example vacancies and voids $[12,18,21,26,30]$, but the influence of transport from different depths to the surface is very often neglected. Fig. 4 shows the $\mathrm{D}_{2}$ release peaks of $\mathrm{W}$-x films implanted with deuterium at 370 and $600 \mathrm{~K}$ with a fluence of $6 \times 10^{24} \mathrm{D} / \mathrm{m}^{2}$ as a function of the real sample temperature. For comparison the $\mathrm{D}_{2}$ release peak of polycrystalline $\mathrm{W}$ measured at identical experimental conditions [32] is also shown. The main release peak of sample W-1 appears around $490 \mathrm{~K}$ and shifts to $510 \mathrm{~K}$ in the samples W-3, W-5 and W-7, and further moves to $520 \mathrm{~K}$ in the sample W-12. Obviously, both the intensity and width of the main release peak increase with increasing film thickness, that means both the amount of retained $\mathrm{D}_{2}$ and the $\mathrm{D}_{2}$ thermal desorption process are affected by film thickness. In addition to the main release peak around $510 \mathrm{~K}$, all spectra of $\mathrm{W}$ films show a shoulder at about $690 \mathrm{~K}$. For the sample W-12 we find a further release peak at about $800 \mathrm{~K}$. The appearance of three desorption peaks suggests the presence of at least three trap energies. These two desorption peaks at 500 and $690 \mathrm{~K}$ are typical $\mathrm{D}_{2}$ release peaks reported for polycrystalline $\mathrm{W}[12,17,21,29-30]$ or $\mathrm{W}[19-20,31]$ films. They are assigned to deuterium trapped in intrinsic defects such as grain boundaries and dislocations. The origin of the desorption peak of sample $\mathrm{W}-12$ at $800 \mathrm{~K}$ is still unclear. The shown spectrum of polycrystalline $\mathrm{W}$ differs significantly from that of the $\mathrm{W}$ films. The polycrystalline sample implanted at $370 \mathrm{~K}$ shows the main $\mathrm{D}_{2}$ release peak at $700 \mathrm{~K}$ and a shoulder peak at around $500 \mathrm{~K}$. Our TPD results indicate that in W films, opposite to the polycrystalline sample, most deuterium is retained in the lower binding energy trap sites. For the $\mathrm{W}$-x films after $600 \mathrm{~K}$ deuterium implantation, the main $\mathrm{D}_{2}$ release peak at $500 \mathrm{~K}$ disappeared and all retained deuterium is desorbed only at temperatures higher than $600 \mathrm{~K}$ with the peak being at about $690 \mathrm{~K}$.

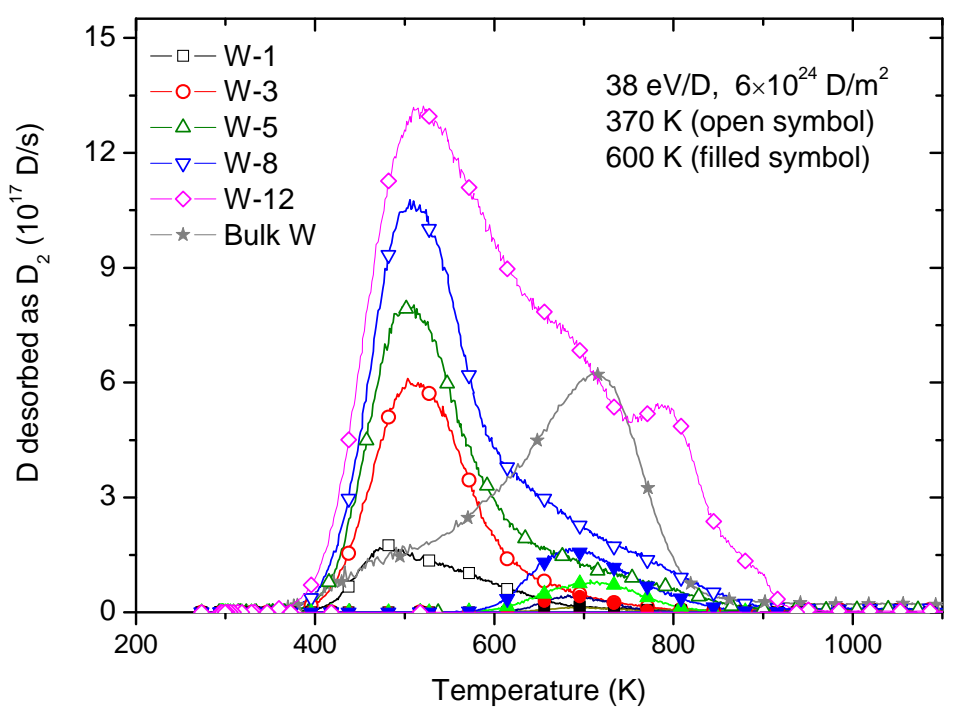

Fig. 4: D2 release spectra from $W$-x layer systems exposed to a fluence of $6 \times 1024 \mathrm{D} / \mathrm{m} 2$ with $38 \mathrm{eV} / \mathrm{D}$ incident energy at a sample temperature of 370 (filled symbol) and $600 \mathrm{~K}$ (open symbol), respectively. In all cases, the oven heating rate is $15 \mathrm{~K} / \mathrm{min}$. 
The main motivation for this $\mathrm{W}$-x film preparation was using this model system to compare the total retained D amount in W measured by TPD and NRA. While this is not possible for bulk targets it is feasible here since all retained deuterium remains within the $\mathrm{W}$ layer and the W layer thickness of all but the W-12 sample is in the range of the NRA information depth of about $8 \mu \mathrm{m}$. For films up to $8 \mu \mathrm{m}$ both TPD and NRA measure the total retained $\mathrm{D}$ amount and the results can be directly compared. One point that should be mentioned is that all TPD measurements were performed during the same week as the NRA measurements to avoid a possible loss of deuterium during additional storage time [24].

The total retained D amounts measured by both TPD and NRA are shown in Fig. 5 as a function of the thickness of the $\mathrm{W}$ coatings. Results are shown for $\mathrm{D}$ exposures $(38 \mathrm{eV} / \mathrm{D}$, $6 \times 10^{24} \mathrm{D} / \mathrm{m}^{2}$ ) at two different target temperatures. Both methods show that the total retained $\mathrm{D}$ amount increases linearly as a function of the W layer's thickness in the whole investigated $\mathrm{W}$ film thickness range. This can be understood assuming that the trap density in our W films is constant and that owing to the high incident fluence applied in all implantations most traps in the $\mathrm{W}$ films are filled. Under these assumptions the retained D amount should increase linearly as a function of the $\mathrm{W}$ layer thickness. Up to $\mathrm{W}$ film thicknesses of $8 \mu \mathrm{m}$, it is obvious that for both cases $(370$ and $600 \mathrm{~K})$ the total retained $\mathrm{D}$ amount determined by these two methods is in excellent agreement. For the samples implanted at $600 \mathrm{~K}$ the deviation of both methods is well within the error bars. The samples implanted at $370 \mathrm{~K}$ show also a very good agreement except the sample W-5, in which the total retained D amount measured by NRA is $12 \%$ lower than amount measured by TPD. But considering the uncertainty of the two measurements this mismatch is still in the range of error bar. Our data clearly shows that both applied methods are able to determine the total deuterium amount in $\mathrm{W}$ films with an uncertainty of less than $10 \%$. But a clear mismatch between TPD and NRA was found for samples W-12 implanted at $370 \mathrm{~K}$. Two such samples were implanted applying the identical plasma conditions as for all other samples. The total retained D amounts in two samples measured by TPD are 252 and $240 \times 10^{19} \mathrm{D} / \mathrm{m}^{2}$, which are 48 to $54 \%$ higher compared to the total D amount measured by NRA. This result had to be anticipated because in this case NRA can only detect the deuterium retained in the top $8 \mu \mathrm{m}$. If we assume that the D concentration in the region from 8 to $12 \mu \mathrm{m}$ is identical to that in the top $8 \mu \mathrm{m}$ then the total D amount in sample $\mathrm{W}-12$ should be $50 \%$ higher than the amount detected by NRA. This is exactly what we observe.

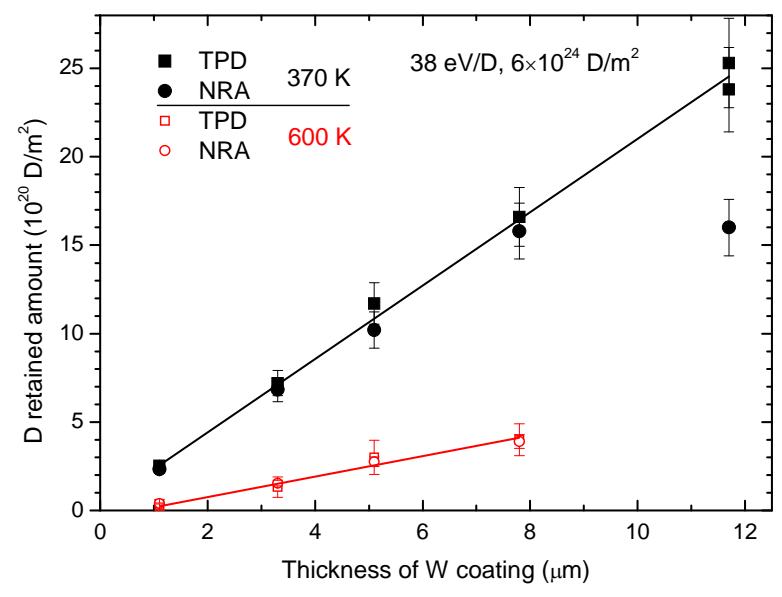

Fig. 5: Total amount of retained deuterium determined by TPD (squares) and NRA (circles) in $W$-x layer systems exposed to deuterium plasma with 38eV/D incident energy and a fluence of $6 \times 1024 \mathrm{D} / \mathrm{m} 2$. Filled and open symbols represent 300 and $600 \mathrm{~K}$ implantation temperature, respectively. 


\section{Summary}

Tungsten films with thicknesses ranging from 1 to $12 \mu \mathrm{m}$ were deposited by magnetron sputtering on silicon substrates. A copper interlayer was used to avoid peeling of the films and to suppress diffusion of deuterium into the silicon substrate. Deuterium was implanted into the samples using the laboratory plasma experiment PlaQ: the deposition parameters were: $38 \mathrm{eV} /$ deuteron, sample temperature 370 and $600 \mathrm{~K}$, and applied fluence $6 \times 10^{24} \mathrm{D} / \mathrm{m}^{2}$. The copper interlayer efficiently blocks the diffusion of deuterium into the silicon substrate. Therefore, deuterium is retained only in the $\mathrm{W}$ films with a well-defined thickness. Using this layer system the total retained D amounts were measured by both TPD and NRA. The deuterium depth profiles determined from the NRA measurements show a D surface peak with about $4 \% \mathrm{D}$ in the top $16 \mathrm{~nm}$, but it has to be kept in mind that the stated thickness of this surface layer is determined by the resolution of the method. Below that surface peak the $\mathrm{D}$ concentration in these films is homogeneous throughout the complete layer for all investigated film thicknesses. The local $\mathrm{D}$ concentration in the depth is about $3 \times 10^{-3}$. This value is a factor of 3-10 higher than the $\mathrm{D}$ concentration in a stress-relieved, polycrystalline tungsten sample implanted under identical conditions. Within the NRA information depth of $8 \mu \mathrm{m}$ the total retained $\mathrm{D}$ amount in the W films measured by both TPD and NRA is in excellent agreement. This means that both methods can be applied to determine total retained deuterium amounts in tungsten with an experimental uncertainty below $10 \%$ for this case. These results can further be taken as indirect confirmation that the used cross section for the $\mathrm{D}\left({ }^{3} \mathrm{He}, \mathrm{p}\right){ }^{4} \mathrm{He}$ nuclear reaction is reliable within $10 \%$.

\section{Acknowledgments}

The stay of P. Wang at Max-Planck Institute for Plasma Physics in Garching was funded through a bilateral agreement between Max-Planck Society and the Chinese Academy of Sciences which is gratefully acknowledged. One of the authors (L. Gao) is grateful to the finical support from MPG-CAS (Max-Planck Gesellschaft \& Chinese Academy of Sciences) Joint Doctoral Promotion Programme. Thanks are further due to Michael Fußeder and Joachim Dorner for help with the RBS and NRA measurements.

\section{References}

[1] R.A. Street, Amorphous Hydrogenated Silicon, Cambridge University, Cambridge, 1993.

[2] J. Robertson, Mat. Sci. Eng. Reports 37 (2002) 129.

[3] T. Schwarz-Selinger, A. von Keudell, W. Jacob, J. Appl. Phys.86 (1999) 3988.

[4] M. Saß, A. Annen, W. Jacob, J. Appl. Phys. 82 (1997) 1905.

[5] G. Federici, C. H. Skinner, J. N. Brooks, et al., Nucl. Fusion. 41 (2001) 1967.

[6] J. Roth, E. Tsitrone, A. Loarte, T. Loarer, et al., J. Nucl. Mater. 390-391 (2009) 1.

[7] R. A. Causey, J. Nucl. Mater. 300 (2002) 91.

[8] W. Jacob, J. Nucl. Mater. 337-339 (2005) 839.

[9] J. Roth, E. Tsitrone, T. Loarer, et al., Plasma Phys. Control. Fusion 50 (2008) 1.

[10] J. Roth, K. Schmid, Phys. Scr. T145 (2011) 014031.

[11] V. Kh. Alimov, J. Roth, Phys. Scr. T128 (2007) 6.

[12] K. Schmid, V. Rieger, A. Manhard, J. Nucl. Mater. 426 (2012) 247.

[13] K. Schmid, U. von Toussaint, Nucl. Instr. Meth. B 281 (2012) 64.

[14] J. F. Ziegler, C. P. Wu, P. Williams, C. W. White, B. Terreault, et al., Nucl. Instr. Meth. B 149 (1978) 19.

[15] V. Kh. Alimov, K. Ertl, J. Roth, J. Nucl. Mater. 290 (2001) 389.

[16] R. Behrisch, M. Mayer, W. Jacob, W. Assmann, et al., J. Nucl. Mater. 281 (2000) 42.

[17] W. M. Shu, E. Wakai, T. Yamanishi, Nucl. Fusion. 47 (2007) 201. 
[18] M. Poon, A. A. Haasz, J. W. Davis, J. Nucl. Mater. 374 (2008) 390.

[19] H. S. Zhou, L. P. Zhao, K. Umstadter, C. P. C. Wong, D. Rudakov, W. Wampler, G. -N. Luo, Fusion Eng. Des. 86 (2011) 325...

[20] V. Kh. Alimov, J. Roth, W. M. Shu, D. A. Komarov, K. Isobe, T. Yamanishi, J. Nucl. Mater. 399 (2010) 225.

[21] O. V. Ogorodnikova, J. Roth, M. Mayer, J. Appl. Phys. 103, (2008) 034902.

[22] E. Salançon, T. Dürbeck, T. Schwarz-Selinger, F. Genoese, W. Jacob, J. Nucl. Mater. 376 (2008) 160.

[23] P. Wang, W. Jacob, L. Gao, T. Dürbeck, J. Nucl. Mater. (2013), http://dx.doi.org/10.1016/j.jnucmat.2013.01.250.

[24] K. A. Moshkunov, K. Schmid, M. Mayer, V. A. Kurnaev, Yu. M. Gasparyan, J. Nucl. Mater. 404 (2010) 174.

[25] R. Dobrozemsky, S. Menhart, K. Buchtela, J. Vac. Sci. Tech. A, 25 (2007) 551.

[26] W. R. Wampler, R. P. Doerner, Nucl. Fusion. 49 (2009) 115023.

[27] M. Mayer, E. Gauthier, K. Sugiyama, U. von Toussaint, Nucl. Instr. Meth. B 267 (2009) 506.

[28] V. Kh. Alimov, M. Mayer, J. Roth, Nucl. Instr. Meth. B 234 (2005) 169.

[29] A. Manhard, K. Schmid, M. Balden, W. Jacob, J. Nucl. Mater. 415 (2011) S632.

[30] M. H. J.'t Hoen, B. Tyburska-Püschel, K. Ertl, M. Mayer, et. al, Nucl. Fusion. 52 (2012) 023008.

[31] O. V. Ogorodnikova, K. Sugiyama, T. Schwarz-Selinger, et. al, J. Nucl. Mater. 419 (2011) 194.

[32] A. Manhard, Ph.D thesis, Deuterium inventory in tungsten after plasma exposure: A microstructural survey. IPP report.

[33] D. Schleußner, H. Maier, P. Franzen, R. Behrisch, et. al, J. Nucl. Mater. 266 (1999) 1296.

[34] B. Landkammer, A. von Keudell, W. Jacob, J. Nucl. Mater. 264 (1999) 48.

[35] A. Manhard, T. Schwarz-Selinger, W. Jacob, Plasma Sour. Sci. Technol. 20 (2011) 015010.

Note: Unfortunately, the information given in the last paragraph of this article is not correct. The contribution of the molecular ions to the total ion flux for standard conditions is: $D_{3}{ }^{+}=94 \%, D_{2}{ }^{+}=$ $3 \%$, and $D^{+}=3 \%$. Correspondingly, the contributions to the total deuteron flux in form of ions are: $97 \%, 2 \%$, and $1 \%$ as expressed correctly in figures 5 and 6 in this reference.

[36] M. Mayer, „SIMNRA User's Guide“, IPP Report Number: IPP 9/113, Max-Planck-Institut für Plasmaphysik, Garching (1997).

[37] K. G. Tschersich, J. Appl. Phys. 87, (2000) 2565.

[38] K. Schmid, A. Manhard, Ch. Linsmeier, A. Wiltner, T. Schwarz-Selinger, W. Jacob, St. Mändl, Nuclear Fusion 50, (2010) 025006.

[39] P. Wang, W. Jacob, M. Balden, T. Höschen, A. Manhard, J. Nucl. Mater. 426 (2012) 277.

[40] V. B. Vykhodets, V. B. Demin, P. V. Geld, Phys. Stat. Sol. A. 34 (1976) 787.

[41] A. Cornu, R. Massot, Compilation of Mass Spectral Data, $2^{\text {nd }}$ Edition, Heyden \& Son, Volume 1, Part A, 1979.

[42] http://www.hidenanalytical.com/index.php/en/cracking-patterns. 